\title{
Three Components of Understanding a Programmer's Manual: Verbatim, Propositional, and Situational Representations
}

\author{
Franz Schmalhofer and Doris Glavanov \\ Universität Heidelberg, West Germany
}

\begin{abstract}
It has recently been proposed that in addition to verbatim and propositional text representations, a reader also forms a cognitive representation of the situations addressed by the text. This theoretical position was supported in three experiments which examined encoding processes, the cognitive products, and retrieval processes of the verbatim, propositional, and situational processing components: The degree of propositional and situational processing was successfully manipulated by varying the subjects' study goals. As a consequence of these differential encoding processes, subjects who studied for text summarization remembered more propositional information while subjects with a knowledge acquisition goal remembered more situational information. It was found that the situational encoding and retrieval processes proceeded faster than the respective propositional processes. In a sentence recognition task, subjects more strongly relied upon situational than propositional information, demonstrating the importance of situational representations in text comprehension. 1986 Academic Press. Inc.
\end{abstract}

While psychologists have investigated in some detail how subjects remember texts (Kintsch, 1974; Kintsch \& van Dijk, 1978; Sachs, 1967; Schank \& Abelson, 1977), in real life texts are often studied with a completely different intention. For example, a student studying a computer science textbook or a car mechanic studying a repair manual is more interested in acquiring knowledge about the respective subject domain as opposed to merely remembering the wording or meaning of a particular text. In order to successfully interact with a computer, the student must develop some mental representation which provides detailed information about the respective system. Such domain knowledge may also

This research was made possible by a travel grant to the first author from Deutsche Forschungsgemeinschaft. We thank Walter Kintsch for his thoughtful comments concerning this work, and Peter Polson. Alice Healy, and the Institute of Cognitive Science at the University of Colorado for permitting us to use the Computer Laboratory for Instruction in Psychological Research (CLIPR) as well as the subject pool. Reprint requests should be addressed to Franz Schmalhofer, Psychologisches Institut, Universität Freiburg, Niemensstr. 10, 7800 Freiburg i. Br., West Germany. be acquired without reading a text, for example through exploring and learning by doing (Anzai \& Simon, 1979). Thus, students' cognitive representations of a particular text and their respective domain knowledge may obviously differ in content.

Recently, van Dijk and Kintsch (1983) have provided several arguments that this distinction between text representations proper and the mental representations of the (real or hypothetical) situations addressed by a text may be very important for psychological theories of text comprehension. Just as philosophy and linguistics distinguish between meaning and reference, van Dijk and Kintsch's discourse theory differentiates between the cognitive representations of a text and the representation of the situations which are addressed by the text. This latter representation which is a representation of a specific domain has been termed the mental or situation model. ${ }^{1}$ It is to be distinguished from verbatim and propositional text representa-

1 The term "situation model" has been used in this paper instead of "mental model" because it better describes the content of information represented. 
tions, which people have been found to construct when reading a text (Kintsch, 1974; Miller \& Kintsch, 1980; Ratcliff \& McKoon, 1978). Unlike a situation model, which may even be constructed in the absence of a text, a propositional text representation is inherently tied to the (reader's perceived) meaning structure of a text. A propositional textbase, which consists of a micro- and macrostructure, thus represents the meaning of a particular text and its gist. A verbatim text representation would be even more closely related to some given text. Since texts can be remembered by their wording (Keenan, MacWhinney, \& Mayhew, 1977) as well as by their meaning (Kintsch, 1974), the psychological relevance of the distinction between verbatim and propositional representations is rather well established.

Although it has been shown that domain knowledge, which is supposedly encoded in a situation model, affects the construction of a text's meaning representation (Spilich, Vesonder, Chiesi, \& Voss, 1979), it also needs to be investigated how (or whether) new domain knowledge can be acquired from studying a text. Van Dijk and Kintsch (1983) have suggested that readers encode such domain knowledge by building (or updating) a situation model when studying a text, in addition to constructing verbatim and propositional text representations. The formation of an appropriate situation model may be of particular importance for utilizing the information presented in the text in order to perform a novel task in the respective subject domain, such as verifying a computer program after having studied a manual (Schmalhofer, 1982).

Since the textbase and the situation model may be constructed by presumably interacting, but nevertheless separate, mental processes, it is expected that subjects who study a text in order to write a summary thereafter (text summarization (TS) subjects), would emphasize propositional text encoding by enhancing the macro- as well as the microprocessing of a text. These subjects should therefore show different reading time patterns than subjects who study the same text in order to acquire knowledge about the respective subject domain (knowledge acquisition (KA) subjects). Whereas the reading times of subjects who read a text for text memory have been shown to be longer for text segments which are located high in the text hierarchy (Cirilo \& Foss, 1980), subjects who study a text for knowledge acquisition are expected to spend relatively more time processing those subordinate paragraphs which present crucial information about the subject domain itself.

As a consequence of these differential encoding processes, TS subjects are predicted to show better propositional text memory, even for micropropositions, than KA subjects. On the other hand, KA subjects should develop a more accurate situational representation than TS subjects. However, if the formation of a situation model is an integral part of the text comprehension processes (Garnham, 1981), even TS subjects could develop stronger situational than propositional representations.

The information retrieval from the two cognitive structures may also be quite different. While a textbase may be searched until some propositions match the propositions of the text sentence, the situational information may be retrieved faster. Supporting evidence has been presented by Reder (1982) who argued that subjects more efficiently judge a test sentence by its plausibility, which is supposedly based upon situational information, than searching memory for an exact propositional match. If, however, TS and KA subjects did not differ by their development of a situation model, no structural differences in the encoding processes, cognitive structures, and retrieval processes would be expected between the two subject groups.

In order to examine the construction of verbatim and propositional text representa- 
tions on the one hand, and representations of the situations referred to by a text on the other, three experiments were performed. Rather than investigating the structure of some fully developed knowledge structure for expert programming, the present study investigates how verbatim, propositional, and situational representations are formed, when people without prior domain knowledge study a programmer's manual for the first time. By instructing subjects to study the manual either for text summarization or for knowledge acquisition, the first experiment investigated differences in the encoding processes of text and situational information, while the second experiment examined differences in the resulting cognitive structures. In order to test the different information retrieval speeds from verbatim, propositional, and situational representations, a speed accuracy trade-off analysis was performed in a third experiment. The three experiments thus investigate the encoding processes, the resulting cognitive structures, and the retrieval processes of three components of text understanding.

\section{EXPERIMENT 1}

In order to investigate the construction of propositional and situational representations in a realistic but controlled setting, subjects who did not know anything about LISP were given part of a LISP programmer's manual to study. This text was well suited for investigating the initial construction of situational representations during text comprehension. The experimental text has a clearly identifiable hierarchical structure which is shown in Figure 1. Whereas the paragraphs at the highest level (Level 1) in the text hierarchy expressed the text's macrostructure, substantive LISP information, which is needed for the construction of a situational representation, was presented at the lower levels of the text hierarchy. Since the most important information for constructing a textbase and a situation model were contained in different paragraphs, differences in the cognitive processing of TS and KA readers can be assessed by comparing the reading times of different text segments. TS readers supposedly emphasize macroprocessing in their construction of a textbase, and should therefore spend more time reading the sentences of the Level 1 paragraphs than the other paragraphs (Cirilo \& Foss, 1980). KA readers, on the other hand, are assumed to emphasize the construction of a situation model and therefore should spend relatively more time on the lower level paragraphs than on the level 1 paragraphs. Consequently, a hierarchical text level by subject group interaction is expected. No prediction, however, can be made, as to which group will show longer overall reading times: If the construction of a macrostructure is relatively more time consuming than the development of a situation model, the overall reading times of $\mathrm{KA}$ readers would be faster and vice versa.

\section{Method}

Subjects. Sixty-four University of Colorado undergraduates, who did not have any knowledge about LISP, participated in the experiment in order to fulfill an introductory psychology course requirement.

Material. A short programmer's manual (742 words) which introduced LISP data representations (atoms and S-expressions) was constructed based upon the first couple of pages of McCarthy, Abrahams, Edwards, Hart, and Levin's LISP I.5 Programmer's Manual (1965). The hierarchical structure of the text thus obtained is shown in Figure 1. In this figure, each paragraph of the text is represented by a node at one of the four different levels in the text hierarchy. The three paragraphs at the highest level (level 1) consisted of an introduction which motivated the reader to study the text, an outline of the material to be presented, and a summary of the presented material. These three paragraphs, which presented the text's macroinformation, occurred as the first, second, and last para- 


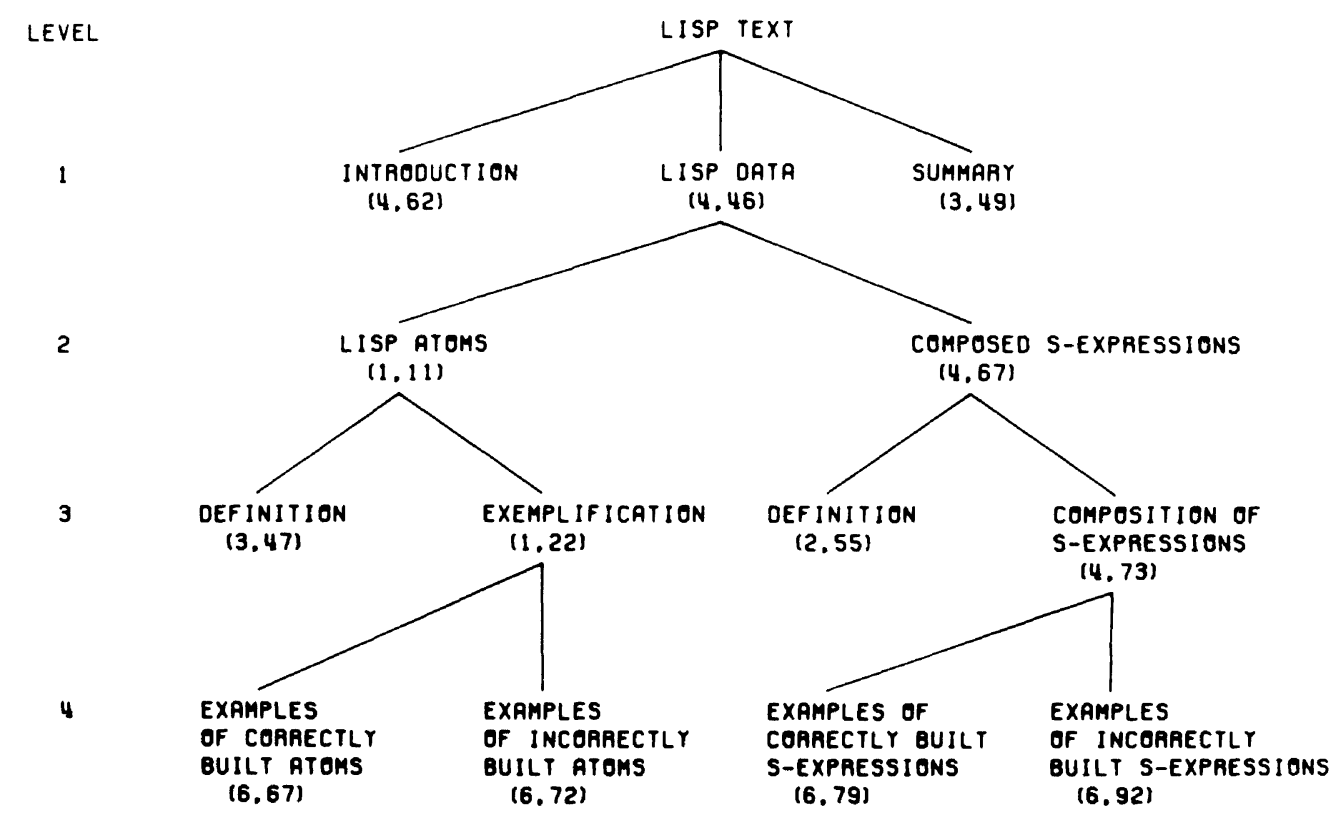

FIG. 1. Hierarchical structure of the paragraphs of the LISP programmer's manual. (The two numbers under the paragraph headlines refer to the number of sentences and the number of words contained in the paragraph, respectively.)

graphs of the text, respectively. The subordinate paragraphs at levels 2 and 3 introduced and elaboratively refined more substantive information about the programming language LISP which should be most useful for the construction of a situation model. The lowest level (level 4) in the text hierarchy presented specific examples of correctly and incorrectly formed LISP data together with brief explanations. ${ }^{2}$

Procedure. The experiment, which was conducted on Visual 200 terminals controlled by the VAX 11/780 computer of the Computer Laboratory for Instruction in Psychological Research at the University of Colorado, was subject paced.

The subjects first read the instructions for the experiment on the terminal screen in order to familiarize themselves with the

\footnotetext{
2 In the text, all five paragraphs on atoms occurred before the paragraphs on S-expressions. Within these two groups of five paragraphs, the paragraphs at the higher levels were presented before the paragraphs at the lower levels and the paragraph to the left, in Figure 1, was presented before the paragraph to the right within a level.
}

mode of presentation of the experimental materials. All subjects were told to study the LISP text in a way that would enable them to do very well on the test that would follow. The only difference in the instructions of the two subject groups concerned the study goal. Half of the subjects were told that the test would involve writing a brief summary of the LISP text (text summarization (TS) readers) and the other half were told that the test would involve a programming task which would consist of writing and verifying LISP expressions (knowledge acquisition (KA) readers).

The LISP text was presented one sentence at a time. In order to indicate the start of a new paragraph, a heading which introduced the topic of the next paragraph was presented before each new paragraph. The headings were presented in all capital letters so as to distinguish them from the text sentences. When subjects had finished studying a sentence, they pressed a button to request the next sentence. They had been informed that they could not return to previously presented sentences and that they should not take any breaks while 
studying the sentences. Sentence reading times were collected by the computer.

\section{Results and Discussion}

A sentence by reading instruction ANOVA revealed that the sentences were read significantly faster by KA subjects (325.8 s) than by TS subjects (406.8 s), $F(1,62)=11.1, M S_{\mathrm{e}}=191.1, p<.005$, and, as would be expected, that the 50 sentences yielded different reading times, $F(49,3038)=64.3, M S_{\mathrm{e}}=16.3, p<.0001$. Since a significant sentence by reading instruction interaction, $F(49,3038)=1.96$, $M S_{\mathrm{e}}=16.3, p<.0001$, was found, KA readers (who read 136.6 words per minute) could not have simply applied the same comprehension processes as TS readers (who read 109.4 words per minute) at a higher speed. Since the TS readers were slower, these results suggest that TS readers were more thoroughly engaged in some relatively time consuming processing component which affected the processing of the 50 sentences differently. KA readers, on the other hand, could have emphasized a different processing component, thus yielding a sentence by subject group interaction and overall reading time differences between TS subjects and KA subjects.

In order to determine which processing components were emphasized by each of the two subject groups, average word reading times were calculated for each level of the text hierarchy. Thus, how the average reading times of the two subject groups depended upon the hierarchical level of the segments in the text could be analyzed. Since the sentences at the lowest text level (Level 4) contained long formulas, Level 4 was excluded from the analysis. In addition to significant group differences, $F(1,62)=9.39, M S_{\mathrm{e}}=0.073, p<$ .005 , and differences between the text levels, $F(2,124)=11.32, M S_{\mathrm{e}}=0.010, p<$ .0001 , a significant interaction was again found, $F(2,124)=3.67, M S_{\mathrm{e}}=0.010, p<$ .05 , indicating structural processing differences between the two subject groups.
The average word reading times for each of the two subject groups and the different text levels are shown in Figure 2. TS readers showed a clear levels effect with the longest word reading times for the highest level in the text hierarchy. Newman-Keuls tests showed that the TS readers' word reading times for the three text levels differed significantly from one another $(p<.05)$. KA subjects, on the other hand, showed the longest word reading times for the second text level, which presented substantial information about the programming language LISP. The Newman-Keuls test yielded a significant difference $(p<.05)$ only between levels 2 and 3 . Thus, unlike TS readers $\mathrm{KA}$ readers did not show a clear levels effect in their reading times. Although it was expected that $\mathrm{KA}$ readers would study the level 2 and level 3 paragraphs approximately equally intensively, a significant difference was observed between the two levels. Since the level 2 paragraphs introduced some basic LISP concepts for the first

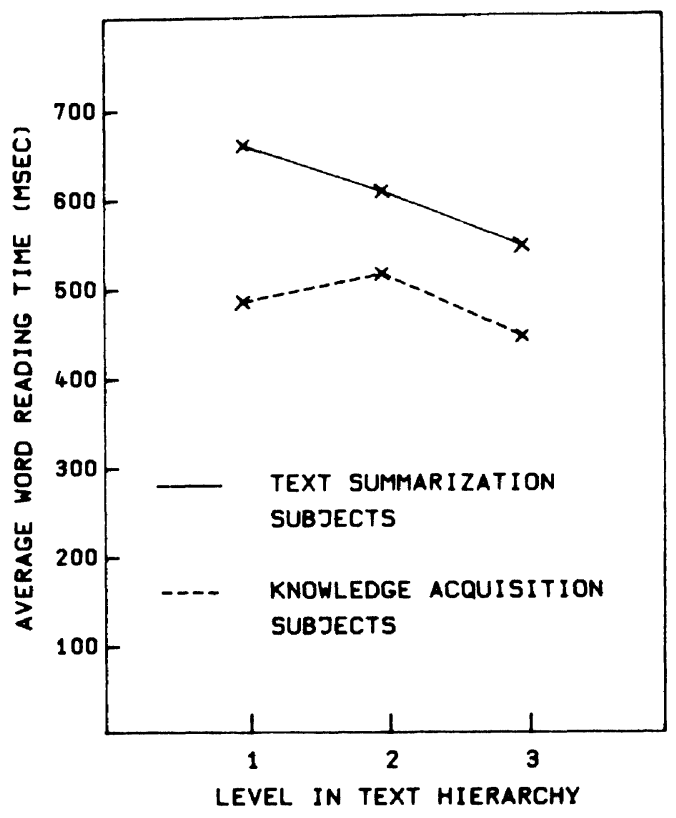

FIG. 2. Average reading times per word (ms) as a function of the level in the text hierarchy for each of the two study instructions (studying in order to write a text summary or studying in order to acquire knowledge about the subject domain). 
time, this result could possibly indicate that the generation of new knowledge elements in a situation model requires more time than further elaborating or updating already existing knowledge elements.

These results suggest that by emphasizing macroprocessing TS readers were more thoroughly engaged in constructing a textbase, whereas $\mathrm{KA}$ readers focused on developing a situation model by processing the more substantive information about LISP. If the textbase and the situation model are indeed the cognitive products of two different processing components, the information processing differences demonstrated in this experiment should also be reflected in the respective cognitive products. In order to test the prediction that $\mathrm{KA}$ readers emphasize the development of a situation model whereas TS readers stress the construction of a textbase, these cognitive products were examined in a second experiment.

\section{EXPERIMENT 2}

If the verbatim memory, the textbase, and the situation model are indeed the cognitive products of verbatim, propositional, and situational processing components, subjects can also utilize the information of these three cognitive products in a sentence recognition task. Although a recognition task asks the subjects to examine whether a sentence occurred in a text literally, in addition to verbatim memory, propositional and situational information may also be utilized for answering this question (Reder, 1982). In order to examine the relative strength of verbatim, propositional, and situational representations, a retrieval model must be specified for the three cognitive structures. It is assumed that during the recognition processing of a sentence, the retrieval results of the three structures are continuously combined (e.g., added) to yield the currently accumulated recognition strength at any point in time. In addition, it is assumed that the accumulated recogni- tion strength determines a subject's recognition decision. By presenting subjects with test sentences which differ only by the contribution of one of the three cognitive structures, the strength of the respective structure may be examined. Four different types of test sentences can be constructed: A sentence may be presented in the form it occurred in the text (O-sentences); it may be paraphrased (P-sentences); its meaning may be changed while preserving its situational correctness ( $M$-sentences); a id its situational correctness could be changed in addition (C-sentences). As shown in Table 1 , the $\mathrm{O}-\mathrm{P}, \mathrm{P}-\mathrm{M}$, and $\mathrm{M}-\mathrm{C}$ sentence pairs differ only by the contribution of the verbatim, the propositional, and the situational representations, respectively.

Under the assumption that the accumulated recognition strengths are normally distributed with equal variances, the strength of verbatim, propositional, and situational representations may be assessed in a signal detection analysis by $d^{\prime}$ (Egan, 1975). The strength of the verbatim representation may be measured as the difference between the response distributions of original and paraphrased sentences. A $d^{\prime}$ value for the verbatim component will be calculated by using the hit rate to $\mathrm{O}$-sentences and the false alarm rate to P-sentences. Similarly, a $d^{\prime}$ value for propositional information may be calculated from the percentage of "yes" responses to Psentences and the false alarm rate to $\mathrm{M}$ sentences. Finally, a $d^{\prime}$ value for situational information may be derived from the percentage of "yes" responses to $\mathrm{M}$ - and $\mathrm{C}$ sentences.

The strengths of the three cognitive structures were examined for text summarization (TS) and knowledge acquisition (KA) readers. Possible trade-off effects of response time and response accuracy were eliminated by determining the asymptote of recognition performance for TS and KA readers with the use of a tapping speed accuracy trade-off paradigm (Wickelgren, Corbett, \& Dosher, 1980). 
TABLE 1

Contribution of Verbatim, Propositional, and Situational Representations to Each of the Fol'r SENTENCE Forms

\begin{tabular}{lcccc}
\hline & \multicolumn{4}{c}{ Test sentence } \\
\cline { 2 - 5 } $\begin{array}{c}\text { Source of } \\
\text { information }\end{array}$ & $\begin{array}{c}\text { Correctness } \\
\text { changed }\end{array}$ & $\begin{array}{c}\text { Meaning } \\
\text { changed }\end{array}$ & Paraphrased & Original \\
\hline Verbatim & - & - & - & + \\
Propositional & - & - & + & + \\
Situational & - & + & + & + \\
\hline
\end{tabular}

Note. The " +" and "- " indicate whether a cognitive structure supplies evidence for a "yes" (old) or " no" (new) recognition decision, respectively.

\section{Method}

Subjects. Sixty-four subjects were recruited from the subject pool used in Experiment 1 .

Material. The programmer's manual prepared for Experiment 1 was also used as the text in this experiment.

Ten affirmative and ten negative sentences on examples of LISP data, which occurred in the level 4 paragraphs of the text, were used to construct the recognition test. Affirmative sentences consisted of two clauses which stated that a given LISP data example is a correctly built type of LISP data (clause 1), with a specific element (clause 2). Negative sentences stated that a given LISP data example is incorrectly formed (clause 1), because of a specific element (clause 2). For each of these original (O-) sentences, three distractors were constructed: Paraphrased (P-), meaning-changed (M-), and correctnesschanged (C-) sentences (see Table 2).
Distractor sentences were created by exchanging words or clauses among the 20 original sentences, so that the distractor sentences consisted only of words and phrases which had occurred in the text. Psentences were constructed by replacing words of the second clause with synonyms. $\mathrm{M}$-sentences were obtained by exchanging the second clauses of $\mathrm{O}$-sentences in such a way that the constructed sentences were still correct with respect to the rules of LISP. For C-sentences, changes of the LISP example which are difficult to recognize, such as deleting or inserting a dot (Schmalhofer, 1982), were applied in addition to exchanging the second clauses; thus the resulting sentences were incorrect with respect to the rules of LISP. In addition, this construction ensured that the LISP data example was consistent with the information presented by the second clause.

The 80 sentences thus obtained were divided into four different versions of the test, with every set containing exactly 5

TABLE 2

An Example of an Original Affirmative and an Oricinal. Negativi: Sinjenci: anid Thitik Risplictivi: Distractors

\begin{tabular}{ccc}
\hline Test sentence & Affirmative & Negative \\
\hline Original & PSY 100 is a legal atom. & BIO.-200 is not a legal atom. \\
that concludes with a number. & because it contains a dot. \\
Paraphrased & PSY 100 is a legal atom, & BIO-200 is not a legal atom. \\
& which ends with a numeral. & sio.-200 is not a legal atom. \\
Meaning-changed & PSY 100 is a legal atom, & since it has a dash. \\
Correctness- & which begins with a letter. & BIO200 is not a legal atom. \\
changed & PSY.100 is a legal atom, & because it concludes with a number.
\end{tabular}


sentences of each form so that each of the 20 original sentences was represented in each version by one of the four different forms (O-, P-, M-, and C-sentences).

Procedure. The entire experiment was controlled by the Vax 11/780 computer. Subjects were told that the experimental session consisted of two independent experiments: A general knowledge test and an experiment on text comprehension. In reality, the general knowledge test served as a practice of the speed accuracy trade off procedure which would later be used in the recognition test.

Both subject groups, TS and KA readers, read the instructions and studied the LISP text, exactly as in Experiment 1. Then they reviewed the text, paragraph by paragraph, until they had studied the text for a total of $9 \mathrm{~min}$. Thus, differences in overall study times between TS and KA readers were eliminated as a confounding factor in the recognition test. Although the overall study time was held constant at 9 min, the individual paragraph reading times, which were controlled by the subjects themselves, could vary.

Recognition test instructions and the recognition test directly followed. In the recognition test, subjects had to determine whether a test sentence had occurred verbatim in the text. In order to provide a warm-up and to increase the proportion of old sentences, the test began with four filler sentences. The actual test consisted of one of the four test versions. Each test version was presented to eight TS and eight KA readers. The order of presentation of the sentences in a test version was newly randomized for each subject.

The procedure for the recognition test was modeled after the tapping speed accuracy trade-off method of Wickelgren et al. (1980). With this method, seven old-new responses were collected for each sentence. The subjects' responses were probed by response signals (tones) which occurred $2 \mathrm{~s}$ apart from each other. For every response signal, a subject had to ei- ther press the "yes" or the "no" button. Contrary to the method of Wickelgren et al., no confidence ratings were collected; that is, for every response signal a subject had to press only one button. Since the subjects gave their first response in the form of a guess, which caused a test sentence to appear on the screen 1 s later, subjects' recognition responses were collected $1,3,5,7,9$, and $11 \mathrm{~s}$ after the presentation of the test sentence.

\section{Results}

Since a performance asymptote was reached approximately $5 \mathrm{~s}$ after the presentation of a test sentence, relative frequencies of "yes" responses were calculated by pooling the responses which had occurred at least $5 \mathrm{~s}$ after the sentence presentation. For TS readers, the relative frequencies of "yes" responses were 0.726 for O-sentences, 0.732 for P-sentences, 0.489 for M-sentences, and 0.165 for C-sentences. For KA readers, the respective frequencies were 0.760 for $\mathrm{O}$-sentences, 0.636 for P-sentences, 0.564 for M-sentences, and 0.161 for $C$-sentences.

For every subject, $d^{\prime}$ scores for the three cognitive representations were calculated. The mean $d^{\prime}$ scores of verbatim, propositional, and situational representations thus obtained for TS and KA readers are shown in Table 3 . A $2 \times 3$ reading goal by cognitive representation ANOVA revealed differences in the strength of the three cognitive representations, $F(2,124)=13.64$, $M S_{\mathrm{e}}=1.58, p<.0001$, as well as a significant interaction between reading goal and cognitive representation, $F(2,124)=3.23$, $M S_{\mathrm{e}}=1.58, p<.05$. The two reading goals did not produce overall performance differences, however, $F<1$. NewmanKeuls tests showed that the interaction effect was due to the two reading goals producing significant effects in different directions for the three memory representations. Newman-Keuls tests furthermore revealed that for TS readers, the strength of the verbatim representation differed significantly 
TABLE 3

$d^{\prime}$ Accuracy Scores of Each Processing Goal for the Three Cognitive Structures

\begin{tabular}{lccc}
\hline & \multicolumn{3}{c}{ Representation } \\
\cline { 2 - 4 } Processing goal & Verbatim & Propositional & Situational \\
\hline Text summarization & -0.10 & 0.84 & 1.15 \\
Knowledge acquisition & 0.38 & 0.25 & 1.42 \\
\hline
\end{tabular}

from the propositional and the situational representation, which were not significantly different. For KA readers, only the situational representation was significantly different from the verbatim and propositional representations, which were about equal.

\section{Discussion}

Since the two subject groups performed about equally well overall in the sentence recognition task, the differences in their performances cannot be explained by one reading goal simply causing a semantically deeper level of text processing (Craik \& Lockhart, 1972) than the other reading goal. Instead, the significant interaction between reading goal and cognitive representation shows that TS and KA readers emphasized different components of text processing. Whereas TS readers developed a better propositional text representation, $\mathrm{KA}$ readers emphasized the construction of a situation model. By demonstrating how the development of cognitive structures depends upon a reader's processing goals, these results provide additional evidence for the distinction between a propositional text representation and a situation model.

Contrary to the experimental predictions, however, KA readers showed better verbatim memory than TS readers. Although this result is surprising, it could be explained by KA readers having studied the example sentences more extensively. Since, the sentences employed in the recognition task provided examples of LISP data, they were more relevant for the construction of a situation model than for the formation of the text's macrostructure. By the end of the study phase, TS and KA readers may therefore have differed with respect to the verbatim information held in working memory that was relevant for the recognition test. The fact that the test phase followed immediately after the study phase in this experiment may thus have caused KA readers to correctly reject about $10 \%$ more paraphrased sentences than TS readers.

\section{EXPERIMENT 3}

In order to eliminate the influence of short-term memory and to further examine the speed with which information is retrieved from the three cognitive structures, an experiment with an interfering task between the study and the test phase was performed. Since in a recognition task a textbase must be searched for a propositional match between the test sentence and textbase, the information retrieval from a textbase has been predicted to be relatively time consuming (Reder, 1982). In comparison, situational information, which may be used to judge a sentence by its plausibility with respect to the situations described by a text, may be accessed more directly without elaborate searching. For example, in a recognition task the information of a situation model may be utilized by translating the test sentence into its situational representation and comparing it with the situation model. With a consistency check (Johnson-Laird, 1980, 1983) a person may thus judge whether the test sentence occurred or could have occurred in the text. Because of these possible differences in the cognitive representations of the textbase and the situation model, it is predicted that 
the information of a situation model is retrieved faster as well as more accurately. This prediction will be tested by examining the speed accuracy trade-off relation of verbatim, propositional, and situational retrieval components in the recognition of sentences.

\section{Method}

Sixty-four subjects from the same subject pool, the same materials, and the same procedure as in Experiment 2 were used. The only difference was that in the present experiment subjects were given an interpolated task between the study and test phase of the text. The interpolated task, which took about $15 \mathrm{~min}$, consisted of a study and test phase with an unrelated text.

\section{Results and Discussion}

Table 4 shows the percentage of "yes" responses for the two subject groups and the four sentence types. The average $d^{\prime}$-retrieval scores of verbatim, propositional, and situational information ${ }^{3}$ which were obtained for the different processing times are shown in Figure 3. For each of the three information retrieval components, separate subject group by processing time $(2 \times 7)$ ANOVAs were performed upon the respective $d^{\prime}$ values. In these analyses, the first level of processing time, which consisted of the subjects' guesses before they even saw the specific test sentence, may be used as a baseline for evaluating the retrieval strengths at 1, 3, 5, 7, 9, and $11 \mathrm{~s}$ after the presentation of the test sentence.

Comparison of subject groups. For verbatim memory, neither reliable main effects nor a significant interaction effect was obtained, demonstrating that, after the interfering task, verbatim memory was very weak or had vanished. Subjects, however, showed reliable memory for propositional information, $F(6,372)=8.59, M S_{\mathrm{e}}=0.67$,

\footnotetext{
${ }^{3}$ Rather than incremental $d$ scores (see Wickelgren et al., 1980), the more widely used $d^{\prime}$ measures were employed throughout this research.
}

$p<.0001$. Since neither subject group nor interaction effects were significant, TS and KA readers showed about the same retrieval performance of propositional information. Situational information was also reliably remembered, $F(6,372)=29.3, M S_{\mathrm{c}}$ $=0.86, p<.0001$. Although no significant group differences were found, a significant interaction, $F(6,372)=2.36, M S_{\mathrm{e}}=0.86$, $p<.05$, demonstrated differences in the retrieval of situational information between TS and KA readers. As seen in Figure 3. these differences are mostly due to $\mathrm{KA}$ readers showing a higher final level of accuracy than TS readers. In summary, the three analyses demonstrated that instead of verbatim information, subjects based their recognition decisions mostly upon propositional and situational information, and that KA readers retrieved more situational information than TS readers.

Comparison of the propositional and the situational retrieval components. In order to compare the relative strengths and the time characteristics of propositional and situational information retrieval, $d^{\prime}$-difference scores between propositional and situational $d^{\prime}$ values were calculaed for the retrieval results at $1,3,5,7,9$, and $11 \mathrm{~s}$ after the presentation of the test sentence. Whereas KA readers retrieved more situational information, $F(5,155)=2.51, M S_{\mathrm{e}}=$ $2.01, p<.05$, a one-way ANOVA did not show a significant processing time effect for TS readers. However, for both subject groups, the relation between the accuracy and the retrieval speed was different for propositional and situational information. KA readers had retrieved significantly more situational than propositional information after $5(t=2.18, p<.05), 7(t=$ $2.81, p<.01), 9(t=2.59, p<.05)$, and 11 $(t=2.65, p<.05) \mathrm{s}$. Even TS readers had retrieved significantly more situational than propositional information at $5 \mathrm{~s}(t=2.31, p$ $<.05)$. Since for TS and KA readers, the maximum difference score occurred at 5 and at $7 \mathrm{~s}$, respectively, rather than at $11 \mathrm{~s}$, it may be concluded that both subject 
TABLE 4

Relative Frequencies of "Yes" Responses at Different Processing Times for the Folr Types of Ttest St:ntinces under Two Reading Goals

\begin{tabular}{|c|c|c|c|c|c|c|c|c|}
\hline \multirow[b]{2}{*}{$\operatorname{Pr}($ yes $)$} & \multicolumn{4}{|c|}{ TS readers } & \multicolumn{4}{|c|}{ KA readers } \\
\hline & $\mathrm{O}$ & $\mathrm{P}$ & M & $\mathrm{C}$ & $O$ & $\mathrm{P}$ & M & C \\
\hline After $1 \mathrm{~s}$ & 0.711 & 0.637 & 0.664 & 0.589 & 0.781 & 0.744 & 0.713 & 0.658 \\
\hline After $3 \mathrm{~s}$ & 0.722 & 0.706 & 0.638 & 0.333 & 0.805 & 0.745 & 0.681 & 0.419 \\
\hline After $5 \mathrm{~s}$ & 0.738 & 0.675 & 0.550 & 0.181 & 0.794 & 0.709 & 0.603 & 0.219 \\
\hline After $7 \mathrm{~s}$ & 0.776 & 0.695 & 0.484 & 0.164 & 0.802 & 0.723 & 0.586 & 0.138 \\
\hline After $9 \mathrm{~s}$ & 0.800 & 0.665 & 0.467 & 0.146 & 0.813 & 0.716 & 0.558 & 0.125 \\
\hline After $11 \mathrm{~s}$ & 0.800 & 0.651 & 0.430 & 0.150 & 0.813 & 0.730 & 0.552 & 0.106 \\
\hline
\end{tabular}

Note. O, P, M, and C refer to original, paraphrased, meaning-changed, and correctness-changed sentences. respectively.

groups retrieved situational information faster than propositional information. As Figure 3 shows, the retrieved propositional information increased rather slowly but constantly with processing time. Situational information, on the other hand, was accessed much faster, and soon reached a performance asymptote.

In order to reveal the differences in the speed and accuracy of propositional and situational information retrieval, the $d^{\prime}$ scores observed at different processing times, $t$, were approximated by a function with speed and accuracy parameters $(\lambda, \beta, \delta)$ :

$d(t)= \begin{cases}\lambda(1-\exp \{-\beta[t-\delta]\}) & \text { for } t>\delta \\ 0, & \text { for } t \leqslant \delta .\end{cases}$

In this function, $\mathrm{d}(t)$ represents the $d^{\prime}$ accuracy at processing time $t$, and $\delta$ specifies the minimum processing time required for achieving a $d^{\prime}$ accuracy different from zero. $\lambda$ specifies the performance asymptote and $\beta$ is the exponential rate parameter, determining the speed with which this asymptote will be approached. This exponential approach to a limit, $\lambda$, has successfully been used for describing memory retrieval processes (Dosher, 1982; Wickelgren et al., 1980).

The parameters of the verbatim, the propositional, and the situational retrieval components were estimated by the
STEPIT minimization program (Chandler, 1965). A least-squares criterion was applied to determine the retrieval functions which best described the observed $d^{\prime}$ values. The number of free parameters was reduced by assuming that the $\delta$-parameter for each of the three retrieval components was the same for both subject groups. Figure 3 shows the retrieval functions thus obtained for verbatim, propositional, and situational information. For the propositional and situational retrieval components, the parameters of these functions are presented in Table 5.

Although the interpretation of the $\lambda-, \beta-$. and $\delta$-parameters of the present model is often problematic because the parameter estimates may show artifactual interactions (Wickelgren et al., 1980), the parameter estimates shown in Table 5 are consistent with the statistical results reported above: A comparison of the $\delta$-parameters indicates that situational information is more readily accessible than propositional information. Also, the $\beta$-parameters are consistent with the hypothesis that situational information is retrieved at a faster rate than propositional information. For reasons of completeness, the parameters obtained for verbatim memory which may be artifactual are also reported. For example, the performance asymptote of verbatim memory of TS readers, $\lambda=0.94$, may be inflated by a low retrieval rate, $\beta=0.0001$, and a large $\delta$-parameter, $\delta=2136 \mathrm{~ms}$. For $\mathrm{KA}$ 

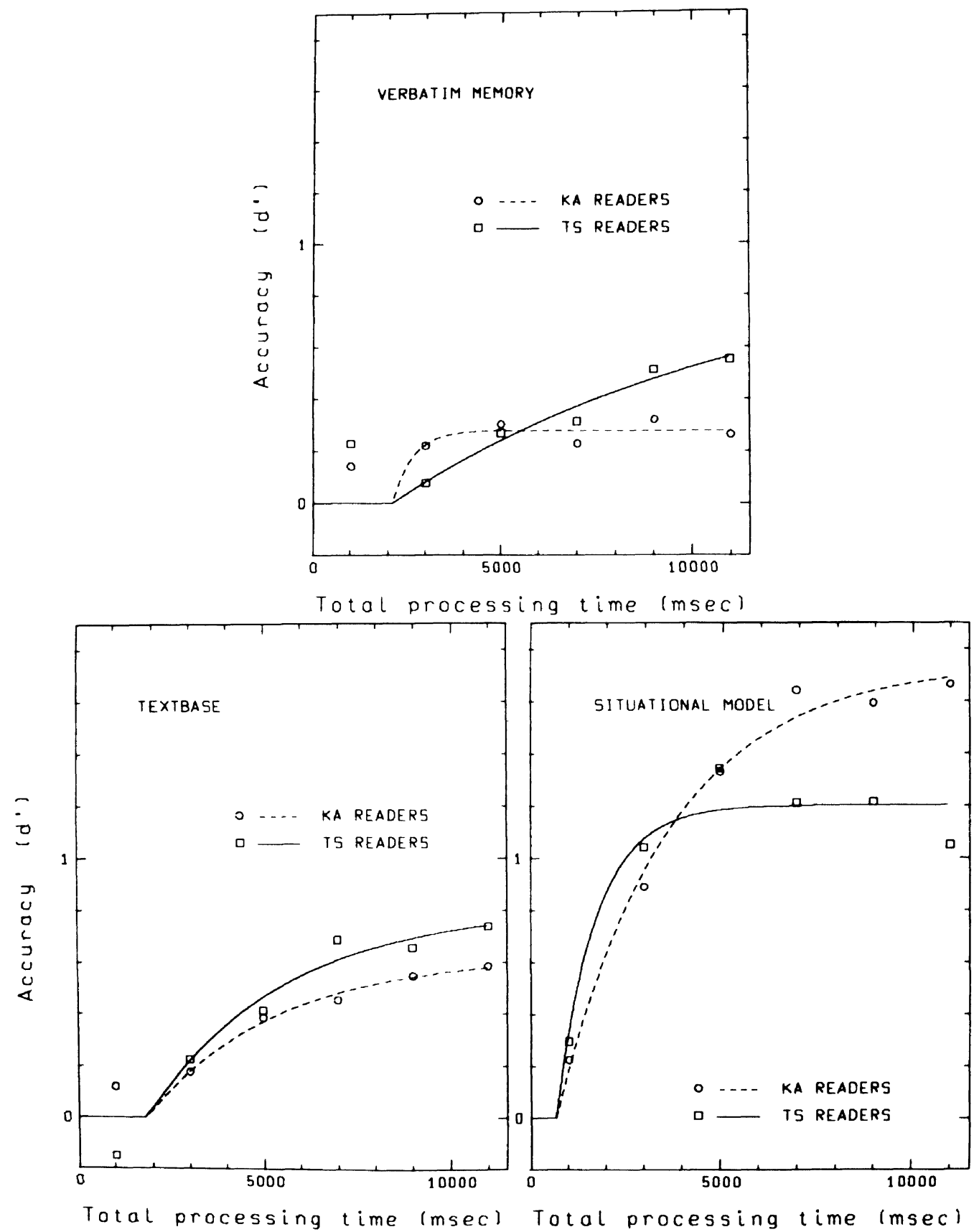

Fig. 3. Accuracy scores $\left(d^{\prime}\right)$ at different processing times for each of the three retrieval components (verbatim memory, textbase, and situation model) and the two study instructions (studying in order to write a text summary (TS) or studying in order to acquire knowledge, (KA)). The smooth curves represent best fitting speed accuracy trade-off functions.

readers, the respective values were $\beta=$ $0.0018, \lambda=0.28$. Since for verbatim memory no significant effect of processing time was obtained by the ANOVA, these parameters should not be interpreted.
In order to evaluate the goodness of fit of the six curves to the 36 data points, the percentage of variance accounted for was determined by a measure which adjusts for the number of parameters estimated from 
TABLE 5

The Values of the Speed and Accuracy Parameters of the Function d( $t$ ) as Estimatid by the: STEPIT Minimization Program for the Propositional and Situational. Rletrifeval. Componints for Eacu Sibilect Grol'p

\begin{tabular}{ccccc}
\hline $\begin{array}{c}\text { Retrieval } \\
\text { component }\end{array}$ & $\begin{array}{c}\text { Subject } \\
\text { group }\end{array}$ & $\begin{array}{c}\text { Intercept } \\
(\mathrm{ms})\end{array}$ & $\begin{array}{c}\text { Retrieval rate } \\
\left(d^{\prime} / \mathrm{ms}\right)\end{array}$ & $\begin{array}{c}\text { Performance } \\
\text { asymptote }\left(d^{\prime}\right)\end{array}$ \\
\hline Propositional & TS & 1847 & $0.00(027$ & 0.81 \\
Situational & KA & & $0.00(0) 29$ & 0.63 \\
& TS & 684 & 0.00095 & 1.20 \\
\hline
\end{tabular}

the data (Reed, 1973). This measure is described by

$$
r^{2}=1-\frac{\sum_{i=1}^{N}\left(x_{i}-\hat{x}_{i}\right)^{2 /(N-K)}}{\sum_{i=1}^{N}\left(x_{i}-\bar{X}\right)^{2 /(N-1)}},
$$

where $N$ is the number of data points $x_{i}, \hat{x}_{i}$ are the respective predicted values, $\bar{X}$ is the grand mean of $x_{i}$, and $K$ is the number of parameters estimated from the data. With an $r^{2}=.94$, the fit between the model and the data can be considered quite good.

When the results of Experiments 2 and 3 are jointly analyzed by a $2 \times 2 \times 3 \times 7$ ANOVA with the factors delay between study and test phase, reading goal, memory representation, and processing time, significant differences were found among the three memory representations, $F(2,248)=$ $20.39, M S_{\mathrm{e}}=6.96, p<.0001$, the different processing times, $F(6,744)=173.75, M S_{\mathrm{e}}$ $=0.22, p<.0001$, the interaction between processing time and memory representation, $F(12,1488)=9.20, M S_{\mathrm{e}}=0.98, p<$ .0001 , as well as a marginally significant memory representation by reading goal interaction, $F(2,248)=2.53, M S_{\mathrm{e}},=6.96, p$ $<.1$. With the exception that the representation by reading goal interaction was only marginally significant, this analysis thus replicated the results of the individual analyses of Experiments 2 and 3.

It appears that the inierpolated task succeeded in clearing short-term memory: While the second experiment only confirmed the predictions about propositional and situational representations, the predicted pattern of results was fully obtained in Experiment 3. Thus, the verbatim memory scores of Experiment 2 may indeed have been influenced by different information being held in working memory at the end of the study phase.

\section{General Discussion}

When readers study a text such as a programmer's manual, three processing components can be distinguished. A reader may process the wording and the meaning of a text as well as the situations addressed by it. For each of the three components, the influence of a reader's processing goal upon the encoding processes, the cognitive products, and the retrieval processes were examined.

Experiment 1 showed that the goal of text summarization (TS) resulted in subjects spending most time processing the text's macroinformation. Under knowledge acquisition (KA) instructions, however, subjects most intensively processed substantive situational information. Whereas Guindon and Kintsch (1984) have shown that subjects perform macroprocesses independent of text summarization instructions, the present results clearly indicate that the intensity of macroprocessing depended upon the two processing goals, which were induced by different reading instructions.

Unlike TS readers, KA readers did not show the longest word reading times for the paragraphs at the highest text level ("levels effect;' Cirilo \& Foss, 1980). The levels ef- 
fect of reading times could thus be a result of the construction of a text's meaning representation and its macrostructure. Whereas TS readers may have constructed the text's macrostructure by applying heuristic comprehension strategies such as micro- and macroprocesses, KA readers, besides constructing the text's microstructure, have emphasized the processing of situational information. Since TS readers studied the text much longer than KA readers, heuristic comprehension processes appear to be more time consuming than the processing of the situations addressed by the text. The encoding time differences between propositional and situational information could be explained by differences in the mode of processing: Whereas the heuristic comprehension processes which construct a propositional textbase (Kintsch \& van Dijk, 1978) are more likely to proceed mostly bottom-up, knowledge acquisition more directly depends upon a reader's prior domain knowledge (Schmalhofer, 1982), and may thus be faster because of the reader's expectations about the situations to be introduced next in the text.

Experiments 2 and 3 showed that TS and KA subjects also differed by the cognitive products constructed during reading. Whereas TS subjects better remembered propositional information than did KA subjects, KA subjects retrieved more situational information. In the third experiment it was found that accessing situational information is faster and proceeds at a higher speed than accessing text information. Even for recognition decisions, situational information is more important than verbatim or propositional text information. In addition to verbatim and propositional text representations, the cognitive representation of situational information is thus an important component when acquiring domain knowledge from studying a text. Although text summarization and knowledge acquisition goals influenced the three processing components in the predicted fashion, the differences among verbatim, propositional, and situational representations were relatively stable for TS and KA goals.

For a technical text such as a programmer's manual, the present study thus provides an intriguing pattern of experimental results for the encoding, memory storage and retrieval of text and situational information which need to be explained by current theories of discourse comprehension. There are basically two ways to account for the differences in the encoding and retrieval speeds and memorability between text and situational information.

One may argue that the difference between text and situational information, which were distinguished from one another by their contents, is just that and does not have any further implication with respect to their representation in memory. Thus only a single (propositional) memory representation would be postulated. Under this assumption, the reading time differences of Experiment 1 could be accounted for in terms of the early Kintsch and van Dijk (1978) model by asserting that the reader's goal, and therefore the schema that formally represents these goals, determines different propositions as relevant for TS and $\mathrm{KA}$ readers. Text summarization readers would consequently emphasize the propositional processing of general statements about LISP, while KA readers would emphasize the processing of the more specific details about LISP. In order to explain the results of Experiments 2 and 3 and in particular how situational information influences subjects' recognition responses, it could be postulated that inferential processes would derive the respective information from a textbase at the time of the test. Such a model, which postulates that two different retrieval strategies operate upon verbatim and propositional memory traces, has been presented by Reder (1982). Supposedly, a direct retrievel strategy searches for an exact match between the wording or propositions of a test sentence 
and verbatim or propositional memory traces. On the other hand, plausibility judgments would calculate the plausibility of the test sentences which had not been inferred during reading from the propositional representation at the time of the test. In addition, Reder postulates that the more consistent a test sentence is with the text, the more likely it is that plausibility judgments would produce a "yes" response in a recognition test: Therefore, more "yes" responses are to be expected for M-sentences than for C-sentences. However, some question remains as to whether such a model could really account for all aspects of the reported data in a straightforward way: If $\mathrm{KA}$ readers formed a propositional text representation which contained more propositions about LISP details, why did these readers then show worse propositional memory for these LISP details (Experiment 2)? Since all test sentences addressed specific details about LISP, KA readers should have shown better propositional memory than TS readers, who supposedly encoded the more general information about LISP.

The theoretical framework set forward by van Dijk and Kintsch (1983) allows for an explanation which assumes that a text and the situations which are addressed by it have their own distinct existence in memory: Whereas the micro- and macrostructure of a propositional textbase represents the meaning of a text and its gist, a situation model presumably represents the (real or fictional) situations addressed by the text. Within this framework the differences in memorability and processing speeds of text and situational information could be explained in terms of organizational differences between the textbase and situation model. Such an explanation would also take into consideration the repeated criticisms of Johnson-Laird and others that propositions by themselves cannot give a complete account of all the relevant aspects of meaning representations in memory. By distinguishing be- tween a propositional textbase and a situation model, the meaning representation of a text can be differentiated from the cognitive representation of the (real or fictional) state of affairs referred to by a text.

In any case, the three experiments, which were performed in order to test some important assumptions of the discourse comprehension strategies described by van Dijk and Kintsch (1983), found that the predictions derived from this theory are at least valid for the particular programmer's manual under study. In order to generalize the results obtained by the method of single cases (Clark, 1973) and to further distinguish between alternative explanations of the reported results, research with different types of texts and different subject populations is needed.

\section{REFERENCES}

Anzal, Y. \& Simon. H. A. (1979). The theory of learning by doing. Psychological Review, 86, $124-140$.

Cirilo, R. K., \& Foss, D. J. (1980). Text structure and reading time for sentences. Journal of Verbal Learning and Verbal Behavior, 19, 96-109.

Chander, P. J. (1965). Subroutine STEPIT: An algorithm that finds the values of the parameters which minimize a given continuous function. Bloomington: Indiana University. Quantum Chemistry Program Exchange.

Clark, H. H. (1973). The language-as-fixed-effect fallacy: A critique of language statistics in psychological research. Journal of Verbal Learning and Verbal Behavior, 12, 335-359.

CRAIK, F. I. M., \& Lo(KHART, R. S. (1972). Levels of processing: A framework for memory research. Journal of Verhal Le'arning and Verbal Behavior. 11, 671-684.

Dosher. B. A. (1982). Effect of sentence size and network distance on retrieval speed. Journal of $\mathrm{EX}$ perimental Psychology: Learning. Memory, and Cognition. 8, 173-207.

Egan, J. P. (1975). Signal detection theory and ROC analysis. New York: Academic Press.

Garnham. A. (1981). Mental models as representations of text. Memory and Cognition, 9, 560-565.

Guindon, R., \& Kintsch, W. (1984). Priming macropropositions: Evidence for the primacy of macropropositions in the memory for text. Journal of Verbal Learning and Verbal Behavior, 23, $508-518$.

JOHNSON-LAIRD, P. N. (1980). Mental models in cognitive science. Cognitive Science, 4, 71-115. 
Johnson-Laird, P. N. (1983). Mental models. Cambridge, MA: Harvard Univ. Press.

Keenan, J. M., MacWhinney, B., \& Mayhew, D. (1977). Pragmatics in memory: A study of natural conversation. Journal of Verbal Learning and Verbal Behavior, 16, 549-560.

KInTsCH, W. (1974). The representation of meaning in memory. New York: Erlbaum.

KintsCh, W. . \& VAN DiJK, T. A. (1978). Toward a model of text comprehension and production. Psychological Review, 85, 363-394.

McCarthy, J., Abrahams, P. W., Edwards, D. J.. HaRT, T. P., \& LEVIN, M. I. (1965). LISP 1.5 programmer's manual. Cambridge, MA: MIT Press.

Miller, J. R., \& Kintsch, W. (1980). Readability and recall of short prose passages: A theoretical analysis. Journal of Experimental Psychology: Human Learning and Memory, 6, 335-354.

Ratcliff, R., \& MCKoOn, G. (1978). Priming in item recognition: Evidence for the propositional structure of sentences. Journal of Verbal Learning and Verbal Behavior, 17, 403-417.

REDER, L. M. (1982). Plausibility judgments versus fact retrieval: Alternative strategies for sentence verification. Psychological Revie'w, 89, 250-280.

ReEd, A. V. (1973). Speed-accuracy trade-off in recognition memory. Science (Washington, D.C.). 181, $574-576$.

SACHS, J. (1967). Recognition memory for syntactic and semantic aspects of connected discourse. Perception \& Psychophysics, 2, 437-442.

SANTA, J. L. (1977). Spatial transformation of words and pictures. Journal of Experimental Psychology: Human Learning and Memory, 3, $418-427$

Schank, R. C., \& Abelson, P. R. (1977). Scripts, plans, goals, and understanding. Hillsdale, $\mathrm{NJ}$ : Erlbaum.

Schmalhofer. F. (1982). Comprehension of a technical text as a function of expertise (Doctoral dissertation. University of Colorado, 1982). University Microfilms International No. 83-(09, 879.

Spilich, G. J., Vesonder, G. T., Chiesi, H. L., \& Voss, J. F. (1979). Text processing of domain-related information for individuals with high and low domain knowledge. Journal of Verbal Learning and Verbal Behavior, 18, 275-290.

van Dijk, T. A.. \& Kintsch, W. (1983). Strategies of discourse comprehension. New York: Academic Press.

Wickelgren, W. A., Corbett, A. T., \& Dosher, B. A. (1980). Priming and retrieval from short-term memory: A speed accuracy trade-off analysis. Journal of Verbal Learning and Verbal Behavior. 19, $387-404$.

(Received February 7, 1984)

(Revision received August 26, 1985) 